\title{
TROPOMI: Solar backscatter satellite instrument for air quality and climate
}

\author{
Johan de Vries $^{* a}$, Erik C. Laan ${ }^{\mathrm{a}}$, Ruud W.M. Hoogeveen ${ }^{\mathrm{b}}$, Rienk T. Jongma ${ }^{\mathrm{b}}$, Ilse Aben ${ }^{\mathrm{b}}$, Huib \\ Visser $^{\mathrm{c}}$, Erik Boslooper ${ }^{\mathrm{c}}$, Heikki Saari ${ }^{\mathrm{d}}$, Marcel Dobber ${ }^{\mathrm{e}}$, Pepijn Veefkind ${ }^{\mathrm{e}}$, Quintus Kleipool ${ }^{\mathrm{e}}$, \\ Pieternel F. Levelt $\mathrm{e}^{\mathrm{e}}$ \\ ${ }^{a}$ Dutch Space BV, Newtonweg 1, P.O. Box 32070, 2303 CP Leiden, The Netherlands \\ ${ }^{\mathrm{b}} \mathrm{SRON}$ Netherlands Institute for Space Research, \\ Sorbonnelaan 2, 3584 CA Utrecht, The Netherlands \\ ${ }^{c}$ TNO Science and Industry, Stieltjesweg 1, 2628 CK, Delft, The Netherlands \\ ${ }^{\mathrm{d}}$ VTT Optical Instruments, Tietotie 3, Espoo, P.O. Box 1000, FI-02044 VTT, Finland \\ ${ }^{\mathrm{e}}$ Royal Netherlands Meteorological Institute (KNMI), \\ Wilhelminalaan 10, 3732 GK De Bilt, The Netherlands
}

\begin{abstract}
TROPOMI is a nadir-viewing grating-based imaging spectrograph in the line of OMI and SCIAMACHY. TROPOMI is part of the ESA Candidate Core Explorer Mission proposal TRAQ and also of the CAMEO satellite proposed for the US NRC decadal study. A TROPOMI-like instrument is part of the ESA/EU Sentinel $4 \& 5$ pre-phase A studies.

TROPOMI covers the OMI wavelengths of 270-490 nm to measure O3, NO2, HCHO, SO2 and aerosols and adds a NIR channel and a SWIR module. The NIR-channel $(710-775 \mathrm{~nm})$ is used for improved cloud detection and aerosol height distribution. The SWIR module (2305 - $2385 \mathrm{~nm})$ measures $\mathrm{CO}$ and $\mathrm{CH} 4$ and forms a separate module because of its thermal requirements.

TROPOMI is a non-scanning instrument with an OMI-like telescope but optimized to have smaller ground pixels (10 $\mathrm{x}$ $10 \mathrm{~km}^{2}$ ) and sufficient signal-to-noise for dark scenes (albedo $2 \%$ ). TROPOMI has the same wide swath as OMI (2600 $\mathrm{km}$ ). In TRAQ's mid-inclination orbit, this allows up to 5 daytime observations over mid-latitude regions (Europe, North-America, China).

The paper gives a description of the TROPOMI instrument and focuses on several important aspects of the design, for example the sun calibration and detector selection status.
\end{abstract}

Keywords: Troposphere, UV-Visible, Trace gas, Climate, Air quality.

\section{INTRODUCTION}

With SCIAMACHY already 5 years succesfully operating on ESA's ENVISAT satellite and a flawless operation of OMI since is launch in 2004 on NASA's EOS-AURA satellite, the authors are working on a follow-up instrument with improvements based on the lessons learned from these instruments, providing a step forward in instrument development and usefulness of its data.

TROPOMI's heritage is from SCIAMACHY and OMI and also other related instruments such as GOME on ERS-2 and METOP and the older very succesful US SBUV and TOMS series instruments. These instruments measure sun radiance backscattered from the Earth atmosphere in the UV - SWIR wavelength range and in nadir viewing geometry. OMI is solely dedicated to nadir viewing whereas SCIAMACHY also includes limb viewing. TROPOMI is focussed on nadir viewing because of its primary goal to be sensitive in the troposphere down to the boundary layer/Earth's surface. For this application nadir viewing is the most suitable technique. For air quality from space and for climate research these measurements play a crucial role for the observations of methane, the ozone layer and related chemical constituents, including clouds and aerosols. 
TROPOMI is part of the ESA Candidate Core Explorer Mission TRAQ (TRopospheric composition and Air Quality) and also of the CAMEO (Composition of the Atmosphere from Mid-Earth Orbit) proposed for the US NRC decadal study. As the ESA Capacity Study ${ }^{1}$, a study on user needs in this field, has recommended to implement a TROPOMI-like instrument, a TROPOMI-like instrument is being investigated in the framework of the ESA/EU Sentinel $4 \& 5$ pre-phase A studies as well.

\section{TROPOMI BASICS}

TROPOMI is an $\mathrm{OMI}^{2,3}$ follow-on instrument in the sense that it is a staring sun backscatter spectrograph which uses its wide field-of-view to image the across-flight swath. It has 2 dimensional detectors which use the first dimension to measure different wavelengths and the second for the swath. The special OMI telescope has a wide viewing angle of 114 degrees, that corresponds to a swath width of $2600 \mathrm{~km}$ on the ground. From sun synchronous polar orbit this allows coverage of the complete globe in one day and at higher latitudes several observations per day as a result of orbit overlap.

The TROPOMI optical channels are defined as given in Table 1.

Table 1 TROPOMI optical channels and their products

\begin{tabular}{|c|c|c|c|l|}
\hline Optical channel & $\begin{array}{c}\text { Full spectral } \\
\text { range }[\mathrm{nm}]\end{array}$ & $\begin{array}{c}\text { Full performance } \\
\text { range }[\mathrm{nm}]\end{array}$ & $\begin{array}{c}\text { Spectral sampling } \\
{[\mathrm{nm}] / \text { oversampling }}\end{array}$ & \multicolumn{1}{|c|}{ Products } \\
\hline UV1 & $270-320$ & $270-310$ & $0.27 / 4$ & $\mathrm{O}_{3}$ \\
\hline UV2 & $295-380$ & $310-370$ & $0.12 / 4$ & $\mathrm{O}_{3}, \mathrm{SO}_{2}, \mathrm{HCHO}^{-}$ \\
\hline VIS & $360-495$ & $370-490$ & $0.18 / 4$ & $\begin{array}{l}\mathrm{NO}_{2} ; \mathrm{O}_{2}-\mathrm{O}_{2} \text { cloud } \\
\text { fraction / pressure }\end{array}$ \\
\hline NIR & $710-775$ & $710-775$ & $0.12 / 4$ & $\begin{array}{l}\text { cloud optical thickness / } \\
\text { pressure / fraction; } \\
\text { aerosol height } \\
\text { distribution, } \mathrm{H}_{2} \mathrm{O}\end{array}$ \\
\hline SWIR & $2305-2385$ & $2305-2385$ & $0.125 / 2$ & CO, $\mathrm{CH}_{4}$ \\
\hline
\end{tabular}

As compared to OMI we added the NIR channel and the SWIR module. The NIR channel enables improvement of the tropospheric results by providing accurate surface albedo, cloud and aerosol information. The SWIR module makes use of the SCIAMACHY heritage and measures $\mathrm{CH}_{4}$ and $\mathrm{CO}$, as indicated in Table 1.

An important improvement compared to earlier instruments is the spatial resolution: the ground pixel of TROPOMI is 10 $\mathrm{x} 10 \mathrm{~km}^{2}$. This value is relaxed to $40 \mathrm{x} 40 \mathrm{~km}^{2}$ for the UV1 channel, because the ground and the lower atmopshere are not visible in this wavelength range and thus larger ground pixels can be used to meet the required signal-to-noise ratio. The high TROPOMI spatial resolution imposes challenging requirements to the optical design, even more so because the high signal-to-noise ratios are to be obtained for ground scenes with an albedo down to $2 \%$ for UV-VIS-NIR, and $5 \%$ for SWIR. These are very dark scenes, whereas OMI was designed for average albedo scenes of $30 \%$.

The TROPOMI design is based on the outcome of two important trade-offs :

- $\quad$ The staring (OMI) concept was selected in favor of the scanning (SCIAMACHY, GOME) concept

- $\quad$ Physically, the UV-NIR and the SWIR channels are placed in separate modules each with their telescope instead of a single unit with single telescope

Other trade-offs are still ongoing, such as the selection of CMOS or CCD detectors for the UV-VIS-NIR instrument and the preferred detector manufacturer both for UV-VIS-NIR and SWIR. With the various available platform opportunities (TRAQ, CAMEO, Sentinel 5), the orbit has not yet been fixed, which implies that we have to keep parallel options open for the sun calibration optics.

The remainder of this paper provides more details on each of these trade-offs. 

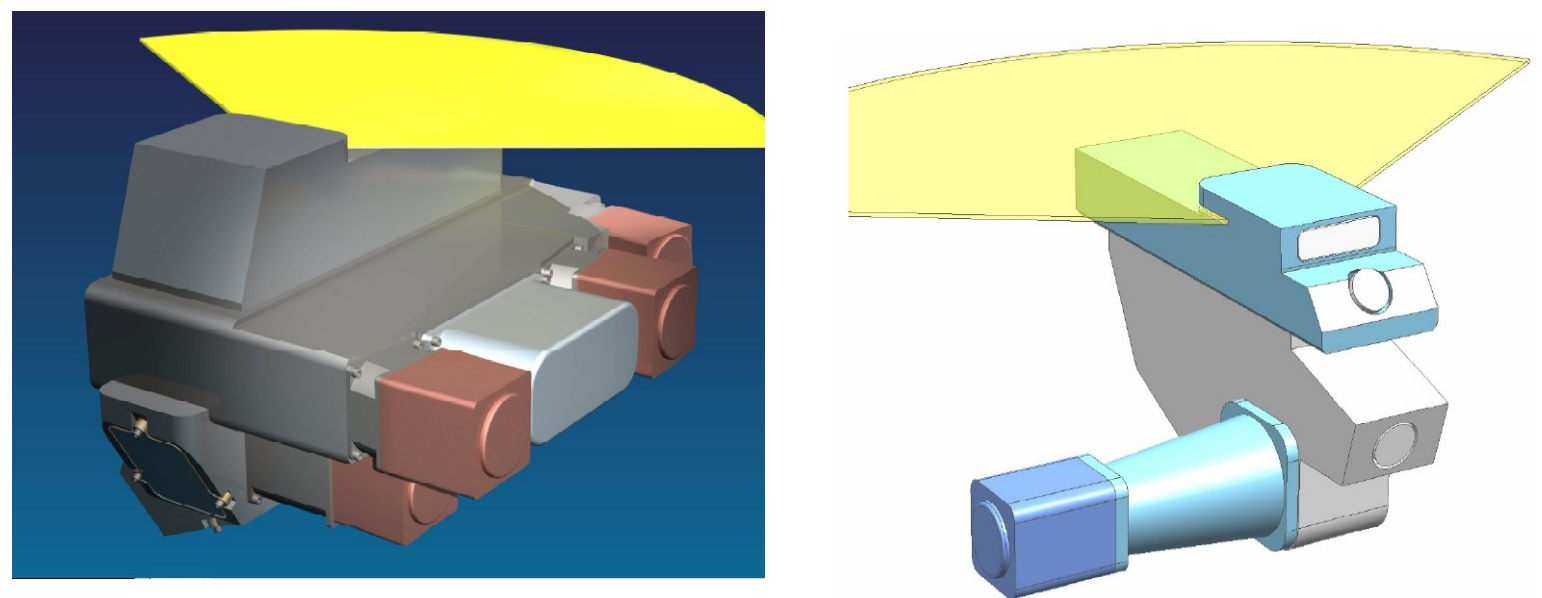

Fig. 1 TROPOMI UV-VIS-NIR module (left) and SWIR module (right). The fields-of-view are indicated in yellow

\section{TRADE OFF BETWEEN SCANNING (SCIAMACHY) AND STARING (OMI) CONCEPTS}

Looking at GOME, SCIAMACHY and OMI, we see a clear trend towards improvement of spatial resolution: GOME provided ground pixels of nominally $320 \times 40 \mathrm{~km}^{2}$, Sciamachy of up to $120 \mathrm{x} 30$, and OMI has pixels of $13 \times 24 \mathrm{~km}^{2}$. Apart from the fact that users tend to ask for data products with improved spatial resolution ${ }^{1}$, a very important benefit is that the fraction of cloud free pixels is increased with smaller ground pixels (see Fig.2).

An important difference between the scanning concept (such as SCIAMACHY, GOME) and staring (such as OMI), is the fact that in the latter all ground pixels in the swath are measured simultaneously and therefore result in longer observation time per ground pixel. The advantage can be appreciated when writing out the equations for the shot noise limited signal-to-noise. The useful signal is expressed as follows:

Where

$\mathrm{S}_{\text {useful }}$

$\mathrm{L}$

$$
S_{\text {useful }}=L \cdot \Delta \lambda \cdot A \cdot \Omega \cdot T_{\text {optics }} \cdot Q E \cdot t_{\exp }
$$

$\Delta \lambda$

A

$\Omega$

$\mathrm{T}_{\text {optics }}$

$\mathrm{QE}$

$t_{\exp }$

useful signal in [electrons]

input radiance [photons $/ \mathrm{cm}^{2}$.sr.s.nm]

spectral sampling distance [nm]

telescope aperture $\left[\mathrm{cm}^{2}\right]$

solid angle [sr]

optics throughput

detector quantum efficiency [electrons/photon]

exposure time [s]

The shot noise limited signal-to-noise is the square root of the useful signal:

$$
S / N_{\text {shotnoise }}=\sqrt{S_{\text {useful }}}
$$




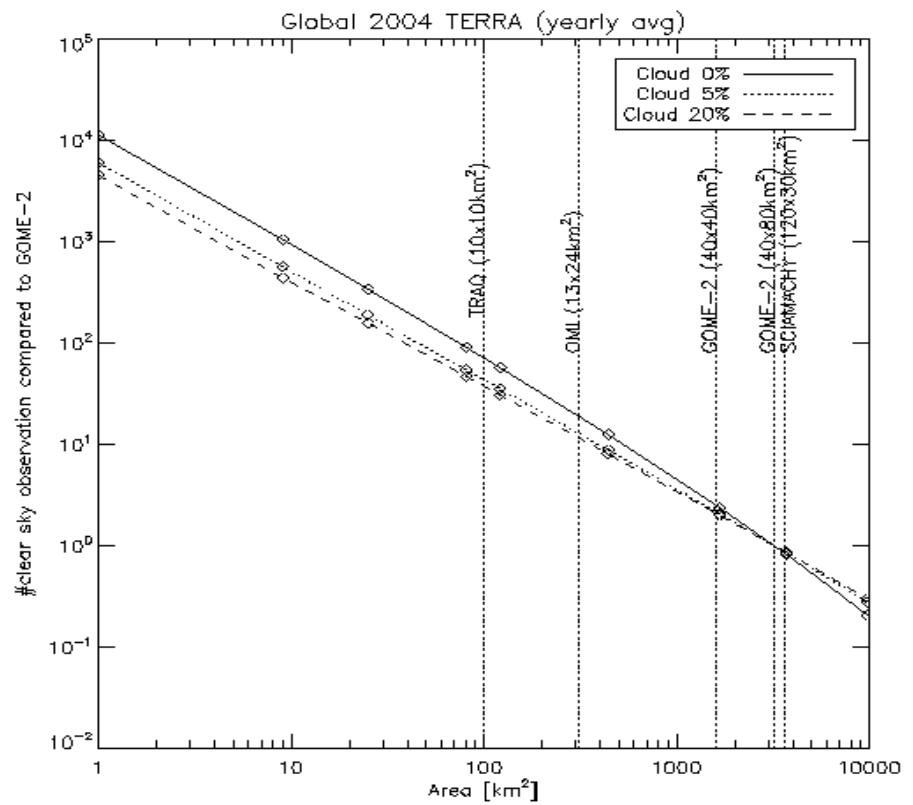

Fig. 2 Relative number of global cloud free pixels as a function of the ground pixel area, for various missions / instruments; there are three curves for different fractions of "cloud free, 8

These equations show that the exposure time directly determines the signal-to-noise and having the full satellite travel time available thus maximizes the signal-to-noise ratios . This is the main advantage of the staring concept, it allows to make use of the complete $t_{\exp }$ for all ground pixels, whereas the scanning concept uses $t_{\text {exp }} / \mathrm{N}$ with $\mathrm{N}$ the number of ground pixels in the swath. On the other hand, the advantage of the scanning concept is that the telescope aperture can be significantly larger as compared to the staring concept. Using the SCIAMACHY aperture of $1.5 \mathrm{~cm}^{2}$ and the OMI aperture of $0.045 \mathrm{~cm}^{2}$, Fig. 2 shows values for A.texp, which is a direct measure for signal-to-noise. Although the curves would shift somewhat if we use other optical designs instead of SCIAMACHY and OMI or if noise details are included, the conclusion is clearly that the shot noise limited signal-to-noise is better for the staring concept for recent user requirements (OMI has 60 ground pixels/swath, TROPOMI will have 140) and that, based on signal-to-noise, it will be beneficial to use the staring concept above approximately 20 pixels / swath.

Signal-to-noise is not the only argument, other important factors are the fact that the staring concept does not require a constantly moving mechanism with potential reliability issues. Furthermore, the staring concept has a straightforward optical location for a polarisation scrambler, whereas the scanning concept does not. A polarisation scrambler has the disadvantage that polarisation information is removed for e.g. aerosol retrievals, but it has the advantage of a more reliable and more accurate radiometric calibration. 


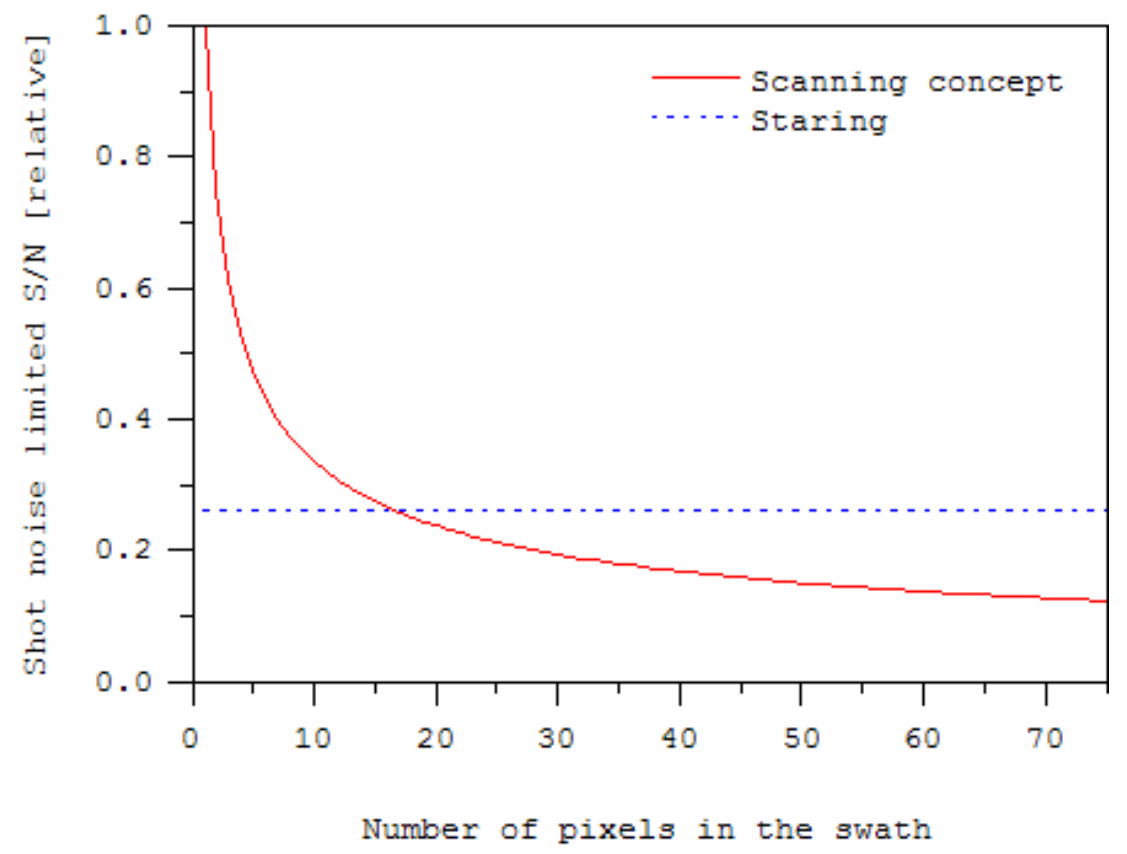

Fig. 3 Relative shot noise limited signal-to-noise from a scanning and a staring concept; the scanning concept has an advantage for smaller numbers of ground pixels in the swath due to its larger telescope aperture; otherwise the staring concept is better because it does not require sharing measurement time between ground pixels

\section{COMBINED OPTICAL BENCH FOR THE UV-NIR AND THE SWIR OR SEPARATED}

Like SCIAMACHY, TROPOMI covers, although with more discontinuities, the wavelength range from the UV to the SWIR. In SCIAMACHY all channels are contained in a single optical bench which is cooled to about $250 \mathrm{~K}$ to have a sufficiently low thermal background signal in the $2.3 \mu \mathrm{m}$ SWIR channel. As the other channels do not require such low operational optical bench temperatures, this approach is not the most efficient in terms of total cooling power. It would be more efficient to thermally isolate the SWIR channel.

The alternative approach is the separation of the UV-VIS-NIR, which typically could operate at room temperature, and the SWIR, which operates at reduced temperature, in independent modules and provide better thermal isolation. This option implies separate telescopes for the two modules. As the telescope is a relatively small element, the main drawback of this option is the additional effort required to precisely align the different telescopes to ensure co-registration between the UV-VIS-NIR and SWIR modules.

Co-registration requires additional alignment effort if separate telescopes are implemented. This is not limited to telescope co-alignment, but extends to the detectors as well. The reason for this is that the co-registration error originating from detector alignment is normally $d_{\text {pixel }} /\left(2 . N_{\text {binning }}\right)$, where $d_{\text {pixel }}$ is the pixel size and $N_{\text {binning }}$ is the number of detector pixels binned into a single ground pixel. This assumes no alignment on detector level, which yielded a (ground-)pixel co-registration error in the OMI case of $24 /(2 \times 8)=1.5 \mathrm{~km}$ or $6.3 \%$ of the ground pixel size of $24 \mathrm{~km}$. In the case of TROPOMI the binning factor is 6 for the UV-VIS-NIR and only 2 for the SWIR. This implies that an additional alignment effort on detector level will be needed. For TROPOMI we would like to have the co-registration error well below $5 \%$. The additional alignment for the separate telescopes is the easier part of this alignment.

Other arguments can be found in polarisation and sun diffuser measurements. As in OMI, the instrument is made insensitive to the incident polarisation by positioning a polarisation scrambler in between the two telescope mirrors. Such a scrambler introduces wavelength dependent features, which increase with wavelength. Implementing separate scramblers for the UV-VIS-NIR and SWIR allows for optimisation to minimise the spectral features in both modules. In 
comparison with the OMI polarisation scrambler the NIR channel adds a new wavelength range, that will need to be optimised as far as spectral features are concerned.

The same is true for the sun measurements via on-board diffusers. In this case the diffuser may introduce spectral and spatial features in the sun measurements. These features increase with wavelength and separation of the UV-VIS-NIR and SWIR modules introduces the freedom of design to optimize this.

The trade-off is summarised in Table 1. The outcome for TROPOMI is to have separate UV-VIS-NIR and SWIR optical benches.

Table 2 Summary of arguments to separate the TROPOMI UV-NIR and SWIR optical benches

\begin{tabular}{lcc}
\hline & $\begin{array}{c}\text { Separated UV-NIR } \\
\text { and SWIR modules }\end{array}$ & All in one module \\
\hline Cooling power & + & - \\
Telescope co-alignment & - & + \\
Polarisation scrambling & + & - \\
Sun diffuser features & + & - \\
\hline
\end{tabular}

\section{DETECTOR SELECTION}

This section is limited to the UV-VIS-NIR module, as for these channels the choice between CCD and CMOS detectors is open and fundamental to the electronics layout. For the SWIR channel, only CMOS detectors are available, and the issue is only to find the best supplier, as discussed by another paper in this conference about the SWIR module ${ }^{4}$.

For the UV-NIR detector selection, the following issues will need to be considered:

- $\quad$ Radiation tolerance

- Data rates and power dissipation

- $\quad$ Science performance

- $\quad$ Development status

These issues are relevant for the development of CMOS type detectors, which provide an alternative to CCD detectors. For TROPOMI much higher internal data rates will be used than for OMI. In addition, the dark current and dark current degradation shall be much lower in TROPOMI than was the case for OMI. This section discusses each of these topics.

CMOS detectors are expected to be more tolerant to in-orbit radiation as compared to CCD detectors ${ }^{5,6}$, but important details need to be considered carefully: both types of detectors use the same light sensitive diodes and the difference has primarily two aspects. The first is the charge transfer process, which is only relevant in CCD detectors and limits detection accuracy of low photon flux levels. Atmospheric applications such as TROPOMI have larger flux levels and are not disturbed too much by charge transfer effects. In particular, Charge Transfer Efficiency (CTE) and its potential degradation is not significant in OMI. The second effect is the increase of dark signals caused by in orbit radiation damage by high energetic protons trapped in the Earth's magnetic field. CCD detectors have much better dark current performance and therefore degradation is more easily detected. In the OMI case, the development of Random Telegraph Signals (RTS) behaviour in pixels that have been hit by a high-energetic proton is particularly disturbing. RTS is an effect originating from the Si bulk material and is observed both in CCD and in CMOS detectors. In the OMI case the effect was dealt with by updating the background correction parameters with a daily frequency.

TROPOMI with its small ground pixels and daily global coverage requires high data rates. In this respect there is a clear advantage for CMOS detectors because their readout noise is essentially independent of the readout rate in contrary to CCD's where the readout noise increases with rate ${ }^{5}$. It is also easier to drive CMOS detectors as compared to CCD's 
which helps especially for the higher rates. Power dissipation can be significantly lower for CMOS detectors which again helps for high data rates.

The science performance of CCD detectors is better as compared to CMOS detectors. Regular CMOS detectors have a limited pixel fill factor which, in our application, effectively reduces the quantum efficiency (QE). However this disadvantage can be avoided by using back-illuminated or hybrid CMOS detectors, in the latter case a separate chip with photodiodes is bonded to a chip with the readout circuitry and a multiplexer. Such CMOS detectors have been produced with good QE for the complete UV-VIS-NIR wavelength range, most importantly also in the UV ${ }^{6}$.

For TROPOMI the other critical performance parameters are pixel-to-pixel non-uniformity (PRNU) and linearity. CCD's outperform CMOS detectors in these aspects and for CMOS detectors this causes increased residual errors originating from the binning designed into TROPOMI. In equation it is as follows :

$$
\sum_{i} S_{i} \cdot P R N U_{i} \neq\left(\sum_{i} S_{i}\right) \overline{P R N U}
$$

Where $\mathrm{S}_{\mathrm{i}}$ is the photon flux for pixel $\mathrm{i}, \mathrm{PRNU}_{\mathrm{i}}$ is the non-uniformity factor for pixel $\mathrm{i}$ and $\overline{P R N U}$ is the pixel averaged PRNU. For non-linearity similar residual errors exist, but in this case it is possible to optimise the detector by choosing between a source follower CMOS design with low power consumption and poorer linearity and a Capacitor Transimpedance Amplifier (CTIA) with higher power and better linearity. For CMOS detectors the non-linearity correction will need to be performed per detector pixel, whereas for CCD's there is one non-linearity correction for all pixels of a detector.

Lastly, the development status as far as high-performance imaging detectors is more advanced for CCD detectors, shown a.o. in the OMI instrument which succesfully uses these detectors. For the TROPOMI project the selection for technology and manufacturer is still to be made.

\section{SUN CALIBRATION}

With the various mission opportunities, the platform or orbit for TROPOMI have not yet been fully fixed. In the TRAQ proposal a mid-inclination orbit is used which has the benefit of allowing multiple observations per day in selected regions of the Earth with a single satellite. CAMEO is in a similar orbit and for the Sentinels 4 and 5, the orbit is still part of a trade-off study.

TROPOMI requires sun calibration measurements at regular intervals in time. In view of the stability of the OMI instrument in its sun-synchronous orbit, these measurements could possibly be several weeks apart but in a more variable mid-inclination it is required to be able to perform sun measurements at a much higher frequency, e.g. every day.

In a polar orbit and with sufficient viewing from the platform, we can have the same approach as for OMI and have the possibility to do a sun measurement for a duration of about 2 minutes at sunset. The measurement time of 2 minutes is useful as it allows the sun incidence angle on the diffuser to change during the measurement period and thereby allow sun diffuser features to average out to a certain extent. In the OMI case it is essential to have amplitude of these spectral features $<10^{-4}$, as required for trace gas retrievals. A similar requirement applies to TROPOMI. The longer sun measurement duration also increases the signal-to-noise ratio considerably.

In the TRAQ mid-inclination orbit and provisional platform concept, it was possible to view the sun during equatorial crossing but with a large range of sun viewing angles. For this option, sun calibration optics were designed where several diffusers are mounted on a wheel and the mechanism to select the diffuser was also used to reduce the sun viewing angles. The latter is important because the radiometric accuracy of the sun diffuser measurements is deteriorated towards larger sun diffuser angles, because of inaccuracies in the on-ground calibration measurements.

For TROPOMI we have decided to have the sun calibration optics as an external module close to the telescope and fixed to the optical bench. This allows us to further detail out the overall optics design while maintaining options for the sun calibration. The currently available options are likely to cover the upcoming orbit / platform opportunities and the OMI heritage provides confidence in the solutions. 


\section{SIGNAL-TO-NOISE RATIOS}

The TROPOMI signal-to-noise requirements are demanding because of the combination of small ground pixels (10 x 10 $\left.\mathrm{km}^{2}\right)$ and reference spectra, for which the signal-to-noises are computed, for very low surface albedos $(2 \%$ in the UVNIR and $5 \%$ in the SWIR). The signal-to-noise requirements have led to a design where the telescope aperture, instrument etendue, detector pixel area and overall optics sizing was carefully tuned to have a reasonably sized instrument, that fits into an envelope of $400 \times 400 \times 450 \mathrm{~mm}^{3}$ or a volume of $72 \mathrm{dm}^{3}$ (most recent values).

The design provides good signal-to-noise values for trace gas retrievals but we still would like to improve the signal-tonoise in the lower wavelength end of the UV2. Fig. 3 shows the UV1 - UV2 overlap where the UV1 signal-to-noise is high due to the larger ground pixel and coarser spectral resolution. We oserve that the signal-to-noise in UV2 close to $310 \mathrm{~nm}$ is below 1000. The UV2 performance can be improved at the cost of the UV1 signal-to-noise by optimising the coating of the channel separation dichroid.

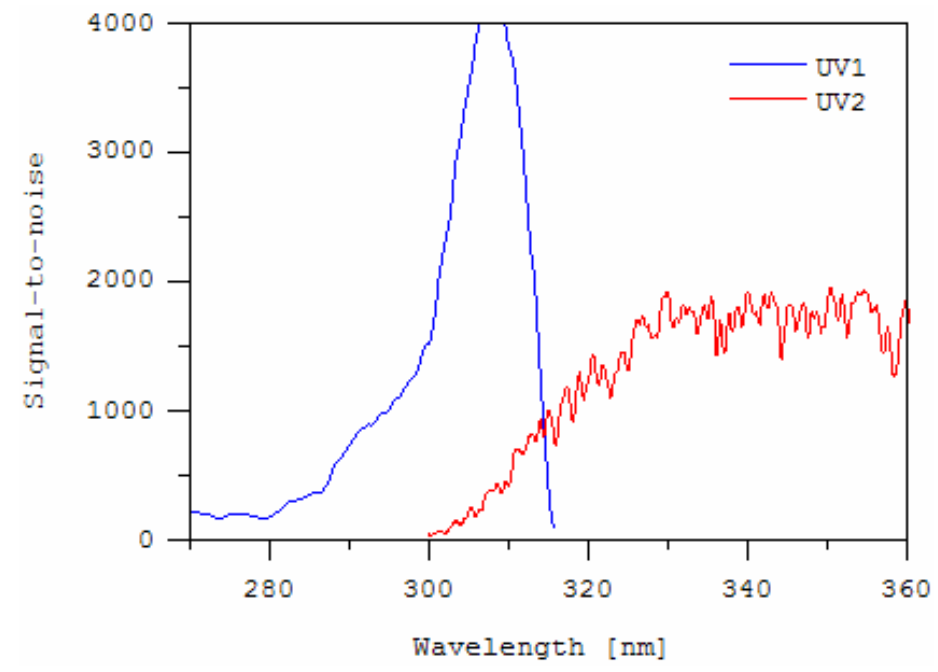

Fig. 4 Signal-to-noise for a $2 \%$ albedo tropical spectrum

\section{C ONCLUSION}

TROPOMI is part of the ESA studies on Earth Explorer Candidate Missions, of the CAMEO study, and is considered for the Sentinels 4 \& 5. A number of important choices and trade-offs in the design of the TROPOMI instrument have been described in detail. The optical design, detector selection, sun calibration and signal-to-noise ratios have been discussed. Final choices on the above mentioned issues will be strongly determined by the exact detailing of the orbit and the satellite platform the TROPOMI instrument in the end will fly on.

\section{REFERENCES}

H. Kelder et al, Capacity Final Report, ESA contract no. 17237/03/NL/GS, October 2005.

P.F. Levelt et al., IEEE Transactions on Geoscience and Remote Sensing, 2005 - 00264

M.R. Dobber et al., IEEE Transactions on Geoscience and remote Sensing, Vol. 44, No.5, May 2006

R.W.M. Hoogeveen et al, this conference, paper 6744A-64

P. Magnan, Nuclear Instruments and Methods in Physics Research A 504 (2003) 199

J. Bogaerts et al, IEEE Transactions on Electron Devices, Vol. 50, No. 1, Jan 2003

Y. Bai et al. Hybrid CMOS Focal Plane Array with Extended UV and NIR Response for Space Application, SPIE San Diego, 3-8 August 2003

8 Krijger, van Weele, Aben and Frey, "Technical note : the effect of sensor resolution on the number of cloud-free observations from space", Atmospheric Chemistry and Physics, 7, 2881-2891 (2007) 